УДК 544.653.2

\title{
ФОТОЭЛЕКТРОХИМИЯ ОКСИДОВ МЕДИ, АНОДНО СФОРМИРОВАННЫХ НА Сu-Zn СПЛАВАХ
}

\author{
(C) 2017 М. Ю. Нестерова, С. Н. Грушевская, А. В. Введенский \\ Воронежский государственный университет, Университетская пл., 1, 394018 Воронеж, Россия, \\ e-mail:sg@chem.vsu.ru
}

Поступила в редакцию 04.07.2017 г.

\begin{abstract}
Аннотация. Анодное окисление $\mathrm{Cu}, \mathrm{Zn}-$ сплавов ( $\alpha$-фаза) в деаэрированном водном растворе $0.1 \mathrm{M} \mathrm{KOH} \mathrm{при} \mathrm{потенциалах} \mathrm{формирования} \mathrm{оксидов} \mathrm{Cu}(\mathrm{I})$ и $\mathrm{Cu}(\mathrm{II})$ исследовано методами хроноамперометрии с синхронной регистрацией фототока, а также измерения фотопотенциала в режиме открытой цепи. Обнаружено, что оксиды меди на сплавах системы Cu-Zn характеризуются $р$-типом проводимости вследствие преобладания акцепторных дефектов. Предварительное селективное растворение сплавов, приводящее к появлению сверхравновесных вакансий в их поверхностной зоне, вызывает увеличение концентрации акцепторных дефектов и уменьшение ширины области пространственного заряда в оксидах.
\end{abstract}

Ключевые слова: медно-цинковые сплавы, оксиды меди, селективное растворение, анодное оксидообразование, фототок, фотопотенциал.

\section{ВВЕДЕНИЕ}

Оксиды металлов играют определяющую роль в анодных и коррозионных процессах, а также находят широкое применение в современных полупроводниковых технологиях. Перспективными материалами в технологиях фотолитического разложения воды, электрокатализа, создания различного рода сенсоров и преобразователей энергии представляются оксиды меди благодаря оптимальному сочетанию таких характеристик, как ширина запрещенной зоны, нетоксичность, низкая стоимость и простота получения.

Потенциостатическое анодное окисление металлов позволяет получать оксидные фазы с контролируемым составом и прогнозируемыми свойствами. Интересная перспектива по модификации свойств оксидных нанопленок открывается при использовании гомогенных сплавов, в частности, сплавов меди с цинком, для которых характерно явление селективного растворения (СР) цинка при контакте со средой [1-3]. Результатом СР является формирование химически измененного поверхностного слоя, представляющего практически чистую, но структурно-разупорядоченную медь. В таком слое концентрация вакансий, образовавшихся за счет преимущественного растворения цинка, заметно превышает равновесную. Степень вакансионной разупорядоченности подложки мо- жет повлиять на кинетику анодного оксидообразования и структурно-зависимые свойства тонких оксидных пленок.

Одними из достаточно доступных и информативных структурно-чувствительных in situ методов исследования полупроводниковых фаз являются фотоэлектрохимические измерения. Теория фотопотенциала $E_{\mathrm{ph}}$ и фототока $i_{\mathrm{ph}}$ развита для объемных полупроводниковых материалов [4-6], при этом размер фазы не влияет на ее фотоэлектрохимические параметры. Однако в [7-10] показано, что в тонких полупроводниковых пленках, толщина которых не превышает ширины области пространственного заряда (ОПЗ), оба параметра уже зависят от толщины оксида:

$$
\begin{gathered}
E_{\mathrm{ph}} \approx-\frac{2 k T}{e} \frac{\eta \Phi_{0}(1-R) \alpha L^{2}}{N_{D} D_{n}} e^{\frac{e\left(E-E_{\mathrm{fb}}\right)}{k T}}, \\
i_{\mathrm{ph}}=e \eta f \Phi_{0}(1-R)\left(1-e^{-2 \alpha L}\right)= \\
=i_{\mathrm{ph}}^{\max }\left(1-e^{-2 \alpha L}\right) \approx 2 \alpha i_{\mathrm{ph}}^{\max } L .
\end{gathered}
$$

Здесь $k$ - постоянная Больцмана $\left(1.38 \cdot 10^{-23}\right.$ Дж/К); $T$ - температура (298 K) ; $e$ - заряд электрона $\left(1.6 \cdot 10^{-19}\right.$ Кл); $\eta$ - внутренний квантовый выход; $\Phi_{0}-$ плотность падающего светового потока; $R$ - коэффициент отражения светового потока от границы оксид/раствор; $\alpha$-показатель поглощения света; $L$ - толщина оксидной пленки; $N_{\mathrm{D}}-$ 
объемная концентрация донорных дефектов; $D_{\mathrm{n}}$ - коэффициент диффузии электронов; $E-$ потенциал формирования оксида; $E_{\mathrm{fb}}-$ потенциал плоских зон; $i_{\mathrm{ph}}^{\max } \quad-$ плотность максимального фототока.

Численная обработка зависимости фототока от толщины оксидной пленки обеспечивает получение ряда ее оптических и структурных параметров. Характер зависимости фотопотенциала от $L$ позволяет сделать определенные заключения о коррозионном поведении металлического электрода, покрытого тонким слоем оксида. Так, увеличение фотопотенциала во времени указывает на коррозионное доокисление материала электрода с утолщением оксидной пленки, тогда как снижение $E_{\mathrm{ph}}$ свидетельствует о преобладании процесса ее коррозионного растворения. Особенно интересен случай, сочетающий отсутствие фотопотенциала в начальный момент после отключения поляризации, его появление и постепенное нарастание с увеличением времени выдержки электрода в растворе. Такое поведение характерно для ситуации, в которой анодное окисление не приводит к образованию оксида, либо его толщина недостаточна для регистрации фотопотенциала. Формирование оксидной тонкопленочной структуры осуществляется лишь после отключения анодной поляризации, по коррозионному механизму.

Цель работы - определить характер влияния уровня вакансионной дефектности поверхностного слоя $\mathrm{Cu}-\mathrm{Zn}$ сплавов на кинетические закономерности анодного формирования тонких пленок оксидов $\mathrm{Cu}(\mathrm{I})$ и $\mathrm{Cu}(\mathrm{II})$, а также их фотоэлектрохимические характеристики.

Выявление такого влияния позволит управлять свойствами оксидных пленок, регулируя концентрацию сверхравновесных вакансий в поверхностной зоне селективно растворяющегося сплава. Кроме того, возможно решение принципиального вопроса о преобладающем механизме образования оксида. Так, само наличие подобной зависимости позволяет сделать выбор в пользу прямого электрохимического окисления металла. В случае преобладания механизма растворения-осаждения появление такой зависимости гораздо менее ожидаемо.

\section{ЭКСПЕРИМЕНТ}

Рабочие электроды с горизонтально ориентированной рабочей поверхностью получены сплавлением меди (99.99 мас.\%) и цинка (99.99 мас.\%) в вакуумированных кварцевых ампулах. Концент- рация цинка $N_{\mathrm{Zn}}$ в исследуемых гомогенных сплавах ( $\alpha$-фаза) составляла $10,15,20$ и 30 ат.\%; соответствующие электроды обозначены как Cu10Zn, Cu15Zn, Cu20Zn и Cu30Zn. Химический состав поликристаллических электродов подтвержден в [11] методом РФЭС, площадь их поверхности составляла $0.53 \div 0.95 \mathrm{~cm}^{2}$. Плотности тока поляризации и фототока рассчитаны на единицу геометрической поверхности; потенциалы приведены в шкале ст.в.э. Подготовка поверхности рабочих электродов, составы растворов и конструкция электрохимических ячеек более детально описаны в [11].

Особенностью ячейки, использованной для измерения фотопотенциала и фототока, является наличие дополнительного вспомогательного платинового электрода. Оксидсеребряный электрод сравнения $(E=0.410 \mathrm{~B})$ приготовлен путем электрохимического окисления серебра в аэрированном 0.1 М КОН в течение 20 мин при $I=5$ мА. В дно ячейки вклеена кварцевая пластинка толщиной 1 мм, через которую проводится импульсное освещение рабочего электрода сверхярким квазимонохроматическим светодиодом «LIGITEK» с длиной волны $\lambda=400$ нм. Ячейка защищена от электромагнитных помех заземленным внешним экраном.

Электрохимические исследования проведены на компьютеризированном потенциостатическом комплексе IPC-Compact производства Института физической химии и электрохимии РАН. После механической и 5-минутной катодной подготовки поверхности сплавов $(E=-0.8 \mathrm{~B})$ проводили потенциостатическую поляризацию в деаэрированном $0.1 \mathrm{M} \mathrm{KOH} \mathrm{при} \mathrm{потенциалах} \mathrm{образования} \mathrm{оксидов}$ $\mathrm{Cu}(\mathrm{I})(E=-0.17 ; 0.00 \mathrm{~B})$ и $\mathrm{Cu}(\mathrm{II})(E=0.10 ; 0.20 \mathrm{~B})$ [11]. В ряде экспериментов перед оксидообразованием сплавы подвергались предварительному СР в деаэрированном $0.01 \mathrm{M} \mathrm{HCl}+0.09 \mathrm{M} \mathrm{KCl}$ при $E=0.1$ В для создания вакансионно-дефектного поверхностного слоя по процедуре, изложенной в [11]. Хотя концентрация сверхравновесных вакансий $N$ в поверхностной зоне таких сплавов достигает 1.3 ат.\% (табл. 1), это практически не влияет

Таблица 1. Концентрация сверхравновесных

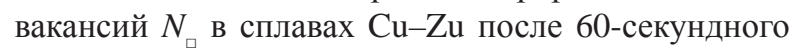
СР в $0.01 \mathrm{M} \mathrm{HCl}+0.09 \mathrm{M} \mathrm{KCl}$ при $E=0.1 \mathrm{~B}$

[Table 1. Concentration of superequilibrium vacancies $N_{\square}$ in $\mathrm{Cu}-\mathrm{Zn}$ alloys after $60 \mathrm{~s}$ selective dissolution in $0.01 \mathrm{M} \mathrm{HCl}+0.09 \mathrm{M} \mathrm{KCl}$ at $E=0.1 \mathrm{~V}]$

\begin{tabular}{|c|c|c|c|c|}
\hline Alloy & Cu10Zn & Cu15Zn & Cu20Zn & Cu30Zn \\
\hline$N_{\square}$, at.\% & 0.08 & 0.82 & 1.32 & 0.20 \\
\hline
\end{tabular}


на потенциалы образования обоих оксидов меди в щелочной среде [11].

Измерение фототока и фотопотенциала осуществляли с использованием потенциостата Compact-2015 Photo Edition, изготовленного в лаборатории возобновляемых источников энергии Санкт-Петербургского Академического университета РАН. Потенциостат характеризуется диапазоном 5-1200 нА по фототоку и 0.5-1500 мкВ по фотопотенциалу. Анодная поляризация с одновременной регистрацией тока поляризации и фо-
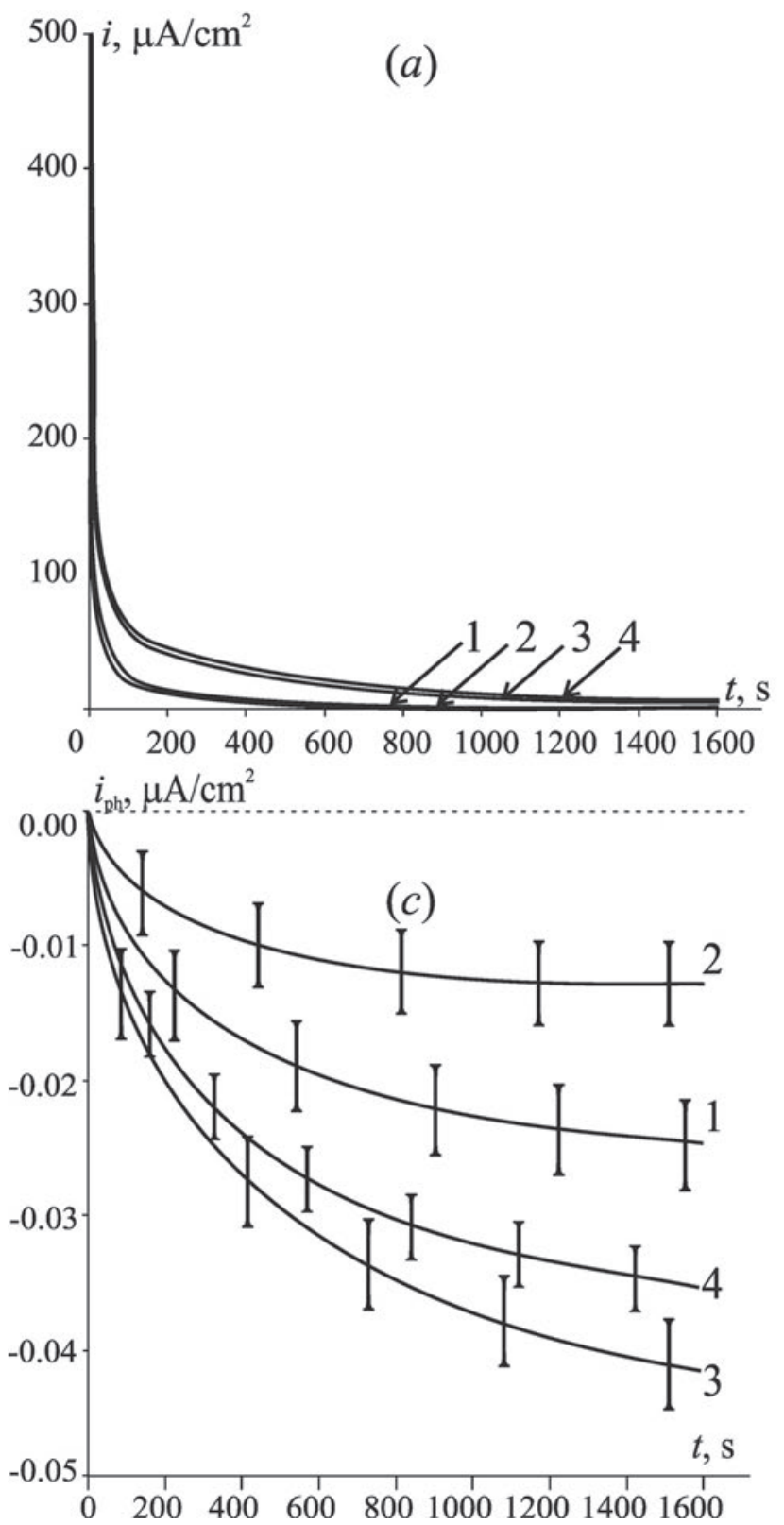

тотока проводилась в течение 30 мин. Измерение фотопотенциала начинали непосредственно после отключения потенциостатической поляризации и проводили в течение 30 мин.

\section{РЕЗУЛЬТАТЫ И ОБСУЖДЕНИЕ}

Кинетика анодного формирования $\mathrm{Cu}_{2} \mathrm{O}$ на сплавах $\mathrm{Cu}-\mathrm{Zn}$. Анодный ток на хроноамперограммах, полученных при потенциалах формирования оксида $\mathrm{Cu}(\mathrm{I})$ на $\mathrm{Cu}, \mathrm{Zn}$-сплавах (рис. 1a), снижается во времени вначале резко, затем более медлен-
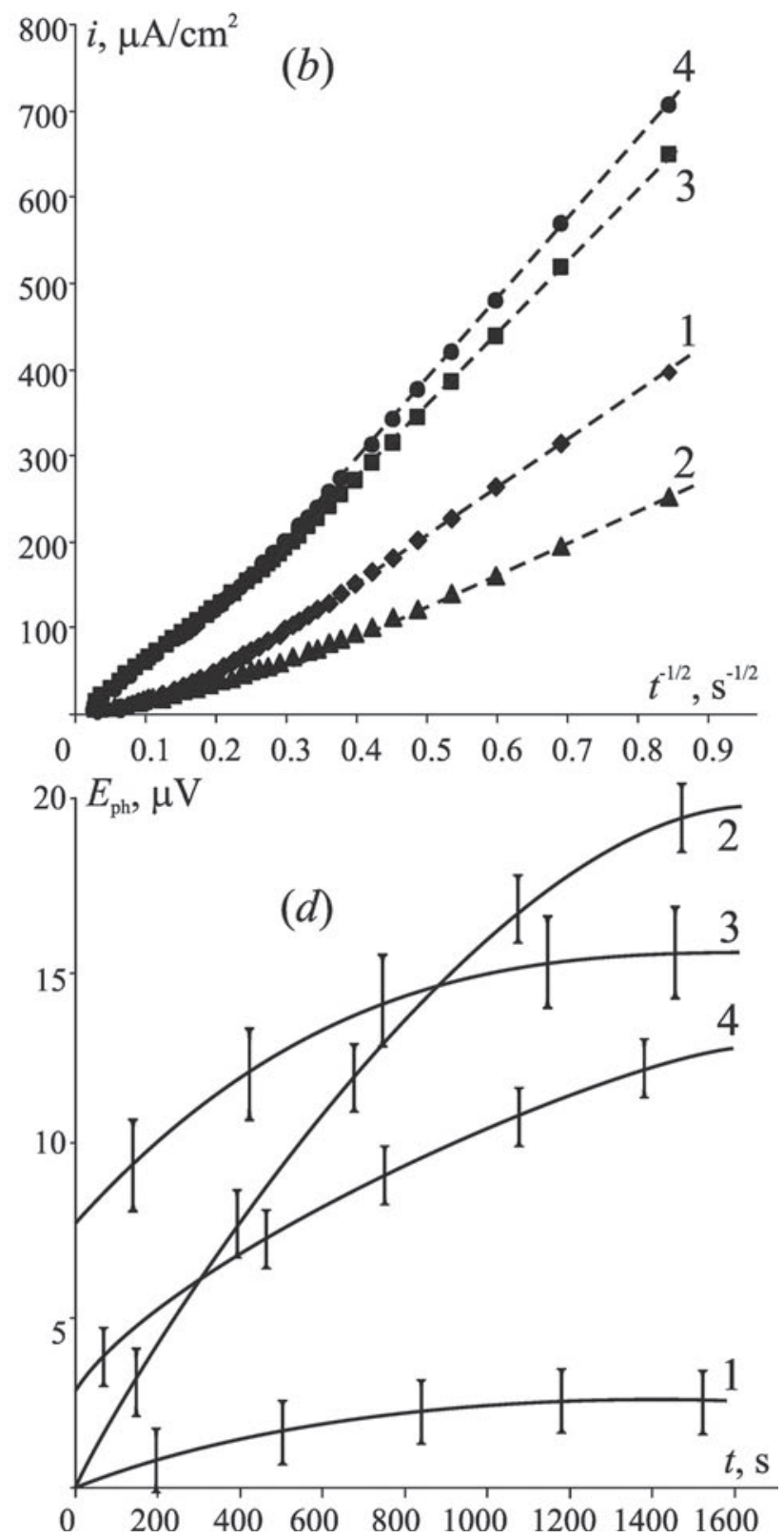

Рис. 1. Хроноамперограммы в обычных (a) и коттрелевских координатах $(b)$, фототок $(c)$ и фотопотенциал $(d)$ на сплавах Cu10Zn (1), Cu15Zn (2), Cu20Zn (3) и Cu30Zn (4) в $0.1 \mathrm{M} \mathrm{KOH} \mathrm{при} \mathrm{E} \mathrm{=-0.17} \mathrm{В}$

[Fig. 1. Current transients in origin $(a)$ and Cottrel coordinates $(b)$, photocurrent $(c)$ and photopotential $(d)$ on Cu10Zn (1), Cu15Zn (2), Cu20Zn (3) and Cu30Zn (4) in $0.1 \mathrm{M} \mathrm{KOH}$ at $E=-0.17 \mathrm{~V}]$ 
но. Плотность тока увеличивается с ростом концентрации цинка в сплаве. В коттрелевских координатах (рис. $1 b$ ) удается выделить линейные участки, свидетельствующие о наличии значительного вклада диффузионных стадий в кинетике процесса. Опираясь на имеющиеся данные по кинетике анодного формирования оксида $\mathrm{Cu}(\mathrm{I})$ на меди [8-10], а также учитывая отсутствие влияния гидродинамического режима на плотность тока, можно сделать вывод о преобладающей роли твердофазного массопереноса в анодном оксидообразовании на всех изученных $\mathrm{Cu}, \mathrm{Zn}$-сплавах. Установление природы диффундирующих частиц и градиента их концентрации требует привлечения сложного комплекса дополнительных исследований, а потому в рамках данной работы определена лишь эффективная константа диффузионного переноса $k_{\text {diff: }}$ :

$$
k_{\text {diff }}=d i / d\left(t^{-1 / 2}\right)=z F \Delta c D^{1 / 2} / \pi^{1 / 2} .
$$

Здесь $z$ - число электронов, $F$ - число Фарадея, $\Delta c$ и $D$ - разница концентраций диффузанта на границах оксидной пленки и его коэффициент диффузии.

Полученные значения $k_{\text {diff }}$ представлены в табл. 2. При потенциалах поляризации $E=-0.17$ и $0.00 \mathrm{~B}$, отвечающих формированию оксида $\mathrm{Cu}(\mathrm{I})$, данный параметр несколько увеличивается с ростом концентрации цинка в сплаве, указывая на некоторое облегчение диффузионного переноса, что предположительно можно связать с увеличением пористости оксидной фазы [12].

При переходе к сплавам, подвергнутым предварительному СР, форма хроноамперограмм при $E=-0.17$ В не меняется, тем не менее, плотность анодного тока и, как следствие, значения $k_{\text {diff }}$ незначительно уменьшаются (табл. 2). Фактически, это свидетельствует о появлении дополнительных затруднений твердофазного массопереноса в оксиде, скорее всего, связанных со снижением дефектности оксидной пленки $\mathrm{Cu}_{2} \mathrm{O}$.

Таблица 2. Эффективные константы диффузионного переноса (мА $\left.\mathrm{c}^{1 / 2} \mathrm{~cm}^{-2}\right)$ формирования оксидов на $\mathrm{Cu}-\mathrm{Zn}$ сплавах в $0.1 \mathrm{M} \mathrm{KOH} \mathrm{без} \mathrm{предварительного} \mathrm{СР} \mathrm{(-)} \mathrm{и} \mathrm{после} \mathrm{него} \mathrm{(+)}$

[Table 2. Effective constants of diffusion transfer $\left(\mathrm{mA} \mathrm{s}^{1 / 2} \mathrm{~cm}^{-2}\right)$ during oxide formation on $\mathrm{Cu}-\mathrm{Zn}$ alloys in $0.1 \mathrm{M} \mathrm{KOH}$ without preliminary selective dissolution (-) and after selective dissolution (+)]

\begin{tabular}{|c|c|c|c|c|c|c|}
\hline \multirow{2}{*}{ Alloy } & \multicolumn{2}{|c|}{-0.17} & 0.00 & \multicolumn{2}{|c|}{0.10} & 0.20 \\
\cline { 2 - 7 } & - & + & - & - & + & - \\
\hline Cu10Zn & 0.50 & 0.10 & 0.80 & 1.00 & 0.80 & 1.50 \\
\hline Cu15Zn & 0.30 & 0.40 & 0.90 & 0.70 & 1.00 & 0.80 \\
\hline Cu20Zn & 0.90 & 0.70 & 1.30 & 1.70 & 3.50 & 2.50 \\
\hline Cu30Zn & 1.10 & 0.55 & 0.90 & 1.60 & 1.80 & 2.00 \\
\hline
\end{tabular}


тервале; абсолютные значения фотопотенциала и характер его зависимости от времени практически не меняются.

Кинетика анодного формирования СиО на сплавах $\mathrm{Cu}-\mathrm{Zn}$. В области потенциалов формирования $\mathrm{CuO}(E=0.10 \mathrm{~B})$ на хроноамперограммах сплавных электродов (рис. 2a) выявляется четкий максимум, отвечающий процессам зародышеобразования; плотности тока при этом весьма высоки. С ростом концентрации цинка в сплаве амплитуда максимума увеличивается, а сам он смеща- ется в область меньших времен. Начальные участки хроноамперограмм спрямляются в коттрелевских координатах (рис. $2 b$ ), указывая на диффузионный контроль процесса. Аналогичная ситуация наблюдается и при $E=0.20 \mathrm{~B}$, но плотности токов немного снижаются, а максимумы становятся более размытыми.

Эффективные константы диффузионного массопереноса при $E=0.10$ и 0.20 В для сплавов с относительно высоким содержанием цинка (Cu20Zn и Cu30Zn) выше, чем для сплавов Cu10Zn и Cu15Zn
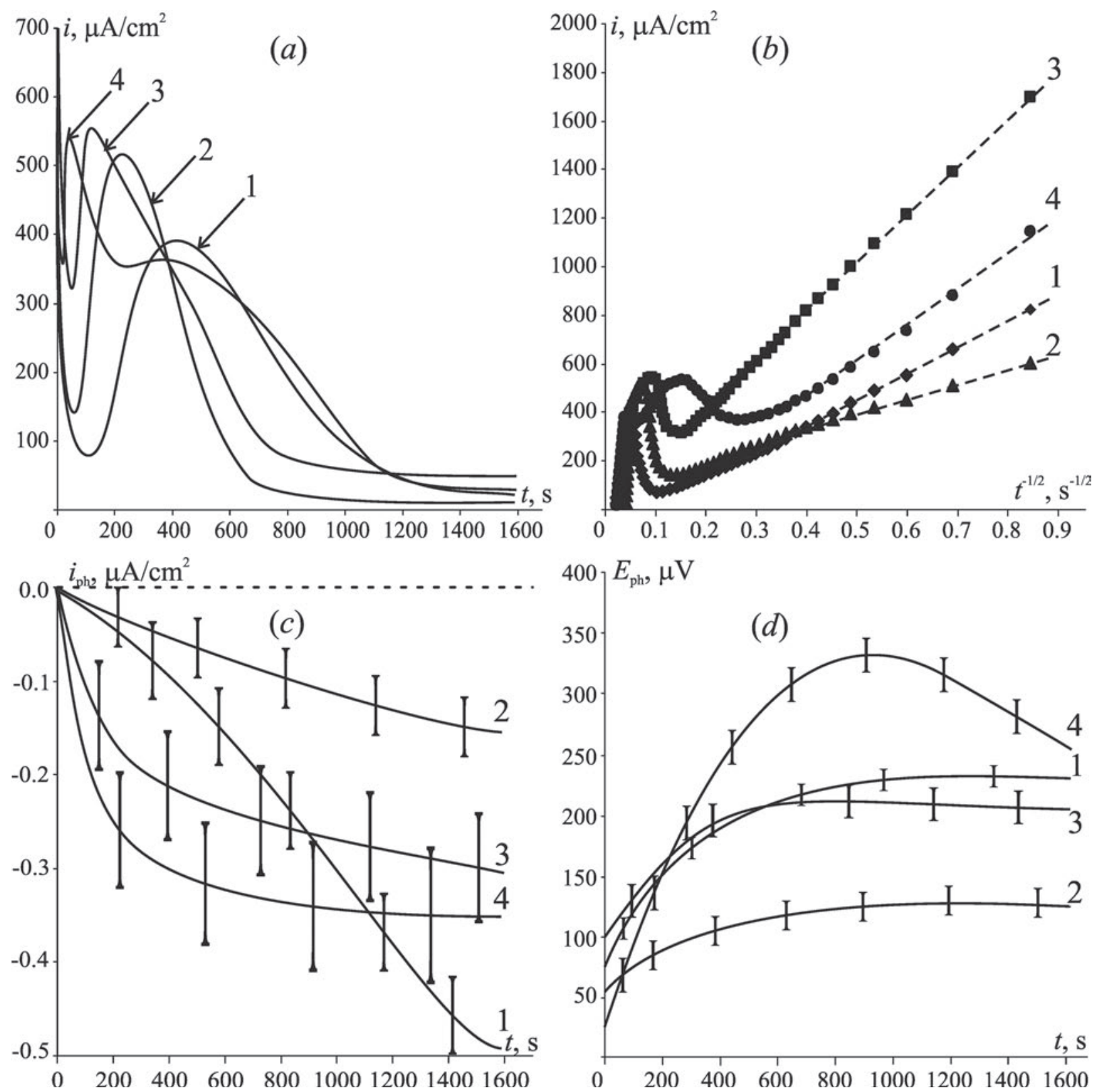

Рис. 2. Хроноамперограммы в обычных (a) и коттрелевских координатах $(b)$, фототок $(c)$ и фотопотенциал $(d)$ в $0.1 \mathrm{M} \mathrm{KOH} \mathrm{при} E=0.10$ В на сплавах Cu10Zn (1), Cu15Zn (2), Cu20Zn (3) и Cu30Zn (4)

[Fig. 2. Current transients in origin $(a)$ and Cottrel coordinates $(b)$, photocurrent $(c)$ and photopotential $(d)$ in $0.1 \mathrm{M} \mathrm{KOH}$ at $E=0.10 \mathrm{~V}$ on Cu10Zn (1), Cu15Zn (2), Cu20Zn (3) and Cu30Zn (4)] 
(табл. 2). Следует отметить некоторое увеличение $k_{\text {diff }}$ с ростом потенциала в широком интервале значений $E$ - от области формирования $\mathrm{Cu}_{2} \mathrm{O}$ до области формирования $\mathrm{CuO}$; эта тенденция особенно ярко проявляется для сплава Cu10Zn. Рост $k_{\text {diff }}$ отражает облегчение твердофазного массопереноca, косвенно указывая на повышение дефектности растущего оксида. Данное заключение согласуется с выводами, сделанными в [11] по значениям токовой эффективности, которые снижаются от 100 до 70 \% при переходе от оксида $\mathrm{Cu}(\mathrm{I})$ к оксиду $\mathrm{Cu}(\mathrm{II})$, что отражает возможность растворения материала электрода через поры оксидной пленки.

На хроноамперограммах сплавов $\mathrm{Cu}-\mathrm{Zn}$ после CР наблюдается увеличение ширины максимумов и их смещение в область больших времен по сравнению с неполяризованными сплавами. В коттрелевских координатах сохраняются линейные участки при малых временах, пока анодный процесс не осложнен зародышеобразованием.

Повышение константы массопереноса $k_{\text {diff }}$ в оксиде $\mathrm{Cu}(\mathrm{II})$ при переходе к сплавам с вакансионнодефектным поверхностным слоем наблюдается при всех $N_{\mathrm{Zn}}$, за исключением $N_{\mathrm{Zn}}=10$ ат.\%. Систематичной зависимости эффективной константы массопереноса от состава сплава выявить не удается. Однако, почти при всех потенциалах, как в отсутствии предварительного СР, так и после него, максимальные значения $k_{\text {diff }}$ характерны для сплава Cu20Zn. Данная ситуация в какой-то степени подтверждает тенденции, ранее выявленные в [11] для зависимостей ряда характеристик (заряда, накопленного в ходе предварительного СР, коэффициента взаимодиффузии компонентов сплава, концентрации сверхравновесных вакансий в его поверхностной зоне и акцепторных дефектов в оксидах меди) от концентрации цинка в объеме сплава. Отмечено, в частности, резкое снижение всех этих величин при переходе от Cu20Zn к сплаву Cu30Zn, обладающему, по данным [13, 14], повышенной стабильностью.

Фотоэлектрохимические характеристики $\mathrm{CuO} \mathrm{на} \mathrm{сплавах.} \mathrm{Амплитуда} \mathrm{катодного} \mathrm{фототока,}$ генерируемого при освещении поляризуемых при $E=0.10$ В сплавов $\mathrm{Cu}-\mathrm{Zn}$, довольно быстро возрастает, после чего стабилизируется (рис. 2c). Исключение составляет сплав Cu10Zn, для которого наблюдается резкий рост фототока. При $E=0.20 \mathrm{~B}$ максимальный фототок характерен для сплава Cu15Zn.

Положительный фотопотенциал, который регистрируется сразу после отключения потенцио- статической поляризации (рис. 2d), быстро растет, а затем принимает стационарное значение или даже немного снижается, как в случае сплава Cu30Zn. Можно предположить, что коррозионное доокисление меди следами молекулярного кислорода сменяется преобладающим растворением оксида в щелочной среде; возможность протекания последнего процесса ранее подтверждена в [15-17].

Заметное увеличение амплитуды фототока, вызванное предварительным СР, регистрируется лишь на сплаве Cu10Zn. Для остальных сплавов характер зависимости фототока от времени, а также его абсолютные значения почти не меняются. Аналогичная ситуация наблюдается и для фотопотенциала.

Зависимость фототока от толщины оксидной пленки. Поскольку фототок и фотопотенциал зависят как от толщины пленки, так и от ее структурных особенностей, то по форме зависимости $i_{\mathrm{ph}}$ и $E_{\mathrm{ph}}$ от времени нельзя судить о влиянии структуры подложки на свойства оксидной пленки. Тем не менее, используя результаты кулонометрии с учетом найденных в [11] значений выходов по току, можно получить зависимости фототока от толщины оксидного слоя $L$. Их численная обработка по (2) позволяет оценить некоторые структурно-чувствительные свойства оксидных пленок.

Пример подобного перестроения приведен для сплава Cu20Zn (рис. 3). С ростом $L$ фототок увеличивается, достигая стационарного уровня, причем

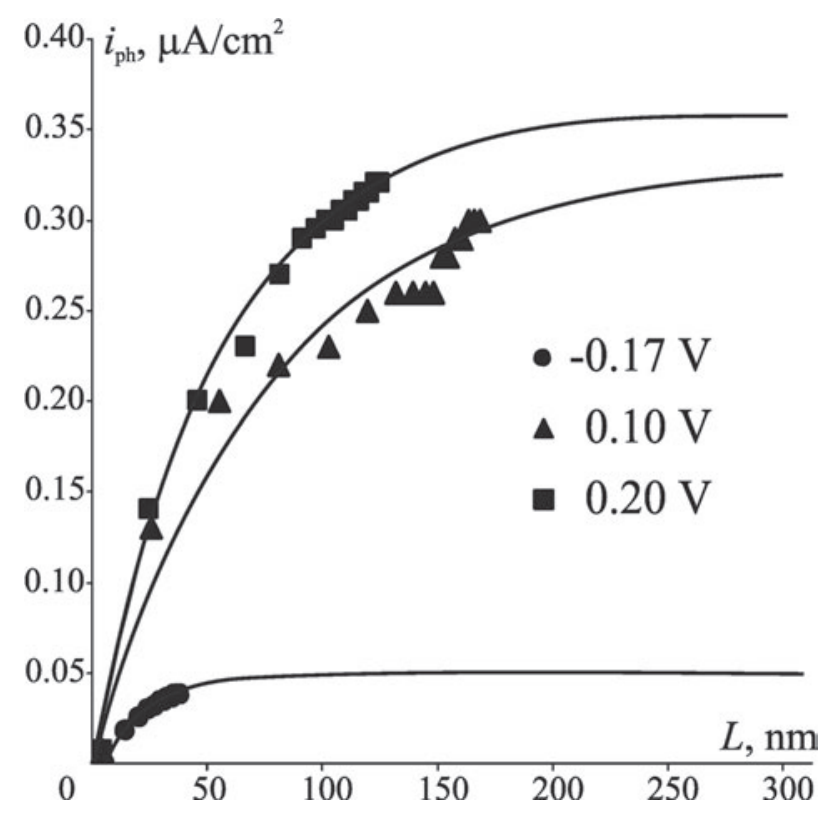

Рис. 3. Зависимость фототока от толщины оксидной пленки, сформированной на Cu20Zn

[Fig. 3. Photocurrent vs. thickness of the oxide film anodically formed on Cu20Zn] 
экспериментальные значения удовлетворительно согласуются с теоретической зависимостью (2).

Комплекс расчетных данных для оксидных пленок, анодно сформированных на сплавах системы $\mathrm{Cu}-\mathrm{Zn}$, не подвергнутых предварительному CP, представлен в табл. 3. В оксиде Cu(II) максимальный фототок и определяющий его параметр $\eta f(1-R)$ больше, чем в оксиде $\mathrm{Cu}(\mathrm{I})$, сформированном при $E=-0.17$ В. Значения максимальных фототоков согласуются с полученными ранее [18] данными для оксида $\mathrm{Cu}(\mathrm{I})$, выращенного на поли-, монокристаллической меди и сплаве Cu4Au.

Толщина оксида, при которой достигается максимальное значение фототока, характеризует ширину области пространственного заряда $W$ :

$$
W=\sqrt{\frac{2 \varepsilon \varepsilon_{0}\left|E-E_{\mathrm{fb}}\right|}{e N_{\mathrm{A}}} .}
$$

Отсюда можно рассчитать концентрацию акцепторных дефектов $N_{\mathrm{A}}$, а через них и дебаевскую длину экранирования $L_{\mathrm{D}}$ :

$$
L_{D}=\sqrt{\frac{\varepsilon \varepsilon_{0} k T}{e^{2} N_{\mathrm{A}}}} .
$$

Здесь $\varepsilon_{0}-$ диэлектрическая постоянная $\left(8.854 \cdot 10^{-12} \Phi / \mathrm{M}\right) ; \varepsilon-$ диэлектрическая проницаемость оксида (10.26 для $\mathrm{Cu}_{2} \mathrm{O}$ и 10.68 для $\mathrm{CuO}$ [19]);
$E$ - потенциал поляризации электрода; $E_{\mathrm{fb}}-$ потенциал плоских зон, составляющий -0.23 и -0.05 В для $\mathrm{Cu}_{2} \mathrm{O}$ и $\mathrm{CuO}$ соответственно [20].

Показатель поглощения света $\alpha$ и концентрация акцепторных дефектов $N_{\mathrm{A}}$ в оксидах на $\mathrm{Cu}, \mathrm{Zn}$-сплавах (табл. 3) в целом меньше, чем на меди [18], а ширина области пространственного заряда $W$, наоборот, больше. При $E=-0.17$ В наблюдается увеличение значений $\alpha$ и $N_{\mathrm{A}}$ с ростом концентрации цинка в сплаве, что указывает на повышение степени отклонения от стехиометрии в оксиде $\mathrm{Cu}(\mathrm{I})$. Ширина области пространственного заряда и дебаевская длина экранирования при этом снижаются. При более высоких потенциалах четкой зависимости этих величин от состава сплава не прослеживается, очевидно, из-за того, что на подслое $\mathrm{Cu}(\mathrm{I})$ начинается формирование оксида $\mathrm{Cu}(\mathrm{II})$.

Аналогичный комплекс расчетных данных получен для оксидов, выращенных на сплавах после предварительного этапа СР (табл. 4). В этом случае максимальный фототок $i_{\mathrm{ph}}^{\max }$ и параметр $\eta f(1-R)$, как правило, больше, чем на сплавах без СР. С ростом концентрации цинка в сплаве показатель поглощения света $\alpha$ и концентрация акцепторных дефектов $N_{\mathrm{A}}$ в $\mathrm{Cu}_{2} \mathrm{O}$ немного увеличиваются, а ширина ОПЗ $W$ и дебаевской области $L_{D}$ уменьшаются. Повышение показателя поглощения и концен-

Таблица 3. Структурные и оптические характеристики оксидов $\mathrm{Cu}(\mathrm{I})$ и $\mathrm{Cu}(\mathrm{II})$, анодно сформированнных на сплавах; $\lambda=400$ нм и $\Phi_{0}=3.04 \cdot 10^{15}$ фотон с см${ }^{-2}$

[Table 3. Structural and optic parameters of $\mathrm{Cu}(\mathrm{I})$ and $\mathrm{Cu}(\mathrm{II})$ oxides anodically formed on alloys; $\lambda=400 \mathrm{~nm}$ and

\begin{tabular}{|c|c|c|c|c|c|c|c|}
\hline Alloy & $E, \mathrm{~V}$ & $i_{\mathrm{ph}}^{\max }, \mu \mathrm{A} / \mathrm{cm}^{2}$ & $\eta f(1-R) \cdot 10^{4}$ & $\begin{array}{c}\alpha \cdot 10^{-5}, \\
\mathrm{~cm}^{-1}\end{array}$ & $W, \mathrm{~nm}$ & $\begin{array}{c}N_{\mathrm{A}} \cdot 10^{-16}, \\
\mathrm{Cm}^{-3}\end{array}$ & $L_{\mathrm{D}}, \mathrm{nm}$ \\
\hline \multirow{4}{*}{ Cu10Zn } & -0.17 & 0.05 & 1.33 & 1.70 & 135.45 & 0.37 & 63.41 \\
\hline & 0.00 & 0.02 & 0.59 & 1.40 & 164.47 & 0.97 & 39.33 \\
\hline & 0.10 & 0.08 & 1.97 & 1.90 & 121.19 & 1.21 & 34.77 \\
\hline & 0.20 & 0.58 & 14.31 & 2.30 & 100.06 & 2.95 & 22.23 \\
\hline \multirow{4}{*}{ Cu15Zn } & -0.17 & 0.04 & 0.90 & 2.08 & 110.70 & 0.56 & 51.83 \\
\hline & 0.00 & 0.05 & 1.19 & 1.70 & 135.45 & 1.42 & 32.39 \\
\hline & 0.10 & 0.08 & 1.87 & 1.39 & 165.65 & 0.65 & 47.52 \\
\hline & 0.20 & 0.80 & 17.62 & 3.96 & 58.15 & 8.74 & 12.92 \\
\hline \multirow{4}{*}{ Cu20Zn } & -0.17 & 0.05 & 0.80 & 2.31 & 99.68 & 0.69 & 46.67 \\
\hline & 0.00 & 0.66 & 10.63 & 2.60 & 88.56 & 3.33 & 21.18 \\
\hline & 0.10 & 0.33 & 5.32 & 0.66 & 348.88 & 0.15 & 100.09 \\
\hline & 0.20 & 0.36 & 5.76 & 0.91 & 253.03 & 0.46 & 56.23 \\
\hline \multirow{4}{*}{ Cu30Zn } & -0.17 & 0.04 & 0.59 & 3.60 & 63.96 & 1.67 & 29.95 \\
\hline & 0.00 & 1.64 & 22.50 & 0.46 & 500.57 & 0.10 & 119.70 \\
\hline & 0.10 & 0.50 & 6.88 & 0.36 & 639.61 & 0.04 & 183.50 \\
\hline & 0.20 & 0.20 & 2.68 & 1.20 & 191.88 & 0.80 & 42.64 \\
\hline
\end{tabular}
$\Phi_{0}=3.04 \cdot 10^{15}$ photon s cm$\left.{ }^{-2}\right]$ 
Таблица 4. Структурные и оптические характеристики оксидов $\mathrm{Cu}(\mathrm{I})$ и $\mathrm{Cu}(\mathrm{II})$, анодно сформированнных на сплавах после предварительного СР; $\lambda=400$ нм и $\Phi_{0}=3.04 \cdot 10^{15}$ фотон $/ \mathrm{c}^{\circ} \mathrm{cm}^{2}$

[Table 4. Structural and optic parameters of $\mathrm{Cu}(\mathrm{I})$ and $\mathrm{Cu}(\mathrm{II})$ oxides anodically formed on alloys after preliminary selective dissolution; $\lambda=400 \mathrm{~nm}$ and $\Phi_{0}=3.04 \cdot 10^{15}$ photon $\left./ \mathrm{s}^{\cdot} \mathrm{cm}^{2}\right]$

\begin{tabular}{|c|c|c|c|c|c|c|c|}
\hline Alloy & $E, \mathrm{~V}$ & $i_{\mathrm{ph}}^{\max }, \mu \mathrm{A} / \mathrm{cm}^{2}$ & $\eta f(1-R) \cdot 10^{4}$ & $\begin{array}{c}\alpha \cdot 10^{-5} \\
\mathrm{~cm}^{-1}\end{array}$ & $W, \mathrm{~nm}$ & $\begin{array}{c}N_{\mathrm{A}} \cdot 10^{-16}, \\
\mathrm{~cm}^{-3}\end{array}$ & $L_{\mathrm{D}}, \mathrm{nm}$ \\
\hline \multirow{2}{*}{ Cu10Zn } & -0.17 & 0.09 & 2.29 & 6.5 & 35.42 & 5.43 & 16.59 \\
\hline & 0.10 & 1.20 & 29.52 & 2.2 & 104.66 & 1.62 & 30.03 \\
\hline \multirow{2}{*}{ Cu15Zn } & -0.17 & 0.04 & 0.74 & 7.5 & 30.70 & 7.23 & 14.37 \\
\hline & 0.10 & 0.10 & 1.99 & 1.4 & 164.47 & 0.66 & 47.19 \\
\hline \multirow{2}{*}{ Cu20Zn } & -0.17 & 0.11 & 1.77 & 7.5 & 30.70 & 7.23 & 14.37 \\
\hline & 0.10 & 0.20 & 3.25 & 0.7 & 328.94 & 0.16 & 94.37 \\
\hline \multirow{2}{*}{ Cu30Zn } & -0.17 & 0.01 & 0.15 & 7.9 & 29.15 & 8.02 & 13.65 \\
\hline & 0.10 & 0.24 & 3.33 & 0.6 & 383.77 & 0.12 & 110.10 \\
\hline
\end{tabular}

трации акцепторных дефектов указывает на увеличение нестехиометрии анодно сформированного оксида $\mathrm{Cu}(\mathrm{I})$.

Таким образом, с ростом вакансионной дефектности поверхностного слоя $\alpha$-латуни, представляющего после СР цинка практически чистую медь, увеличивается и уровень структурной разупорядоченности анодно сформированного оксида $\mathrm{Cu}(\mathrm{I})$. Наличие такого влияния позволяет сделать вывод о доминировании прямого механизма окислении в процессе анодного формирования оксида $\mathrm{Cu}(\mathrm{I})$. Показательно, что для оксида $\mathrm{Cu}(\mathrm{II})$ четкой зависимости структурных параметров от концентрации цинка в латуни и уровня вакансионной дефектности ее поверхностного слоя сплава выявить не удается. Следовательно, в процессе анодного формирования оксида $\mathrm{Cu}(\mathrm{II})$ скорее преобладает механизм растворения и последующего осаждения из пересыщенного по катионам $\mathrm{Cu}^{+}$приэлектродного слоя.

\section{ВЫВОДЫ}

1. Анодное формирование оксидов $\mathrm{Cu}(\mathrm{I})$ и $\mathrm{Cu}(\mathrm{II})$ в деаэрированной щелочной среде на сплавах системы Cu-Zn с концентрацией цинка 10; 15; 20 и 30 ат.\% контролируется твердофазным массопереносом. Эффективная константа диффузионного переноса $k_{\text {diff }}$ увеличивается с ростом потенциала поляризации и концентрации цинка в сплаве. Повышение вакансионной дефектности поверхностного слоя сплава в результате предварительного селективного растворения цинка ведет к снижению константы $k_{\text {diff }}$ в оксиде $\mathrm{Cu}(\mathrm{I})$, но ее росту в оксиде $\mathrm{Cu}(\mathrm{II})$.

2. В оксидах $\mathrm{Cu}(\mathrm{I})$ и $\mathrm{Cu}(\mathrm{II})$ на сплавах при импульсном УФ-освещении генерируется катодный фототок и положительный фотопотенциал, что подтверждает $p$-тип проводимости оксидных пленок. Наличие зависимости фототока от времени указывает на малые толщины оксидных пленок, не превышающие ширины области пространственного заряда полупроводниковой фазы. Максимальный фототок в целом увеличивается с ростом потенциала поляризации и концентрации цинка в сплаве.

3. Рост абсолютных значений фотопотенциала во времени после отключения поляризации отражает возможность протекания коррозионного доокисления меди следами молекулярного кислорода, присутствующего в щелочной среде. По достижении определенного значения ( 300 мкВ) рост фотопотенциала сменяется его спадом. Последнее, по-видимому, обусловлено преобладанием процесса растворения оксидной пленки над ее формированием.

4. Для анодно сформированного оксида $\mathrm{Cu}(\mathrm{I})$ показатель поглощения света $\alpha$, а также концентрация акцепторных дефектов $N_{\text {A }}$, полученные по зависимостям фототока от толщины пленки, увеличиваются с ростом концентрации цинка в $\mathrm{Cu}, \mathrm{Zn}-$ сплаве, тогда как ширина области пространственного заряда $W$ и дебаевская длина экранирования $L_{\mathrm{D}}$ при этом снижаются. Для оксида $\mathrm{Cu}(\mathrm{II})$ четкой зависимости данных параметров от состава сплавов $\mathrm{Cu}-\mathrm{Zn}$ не выявлено.

5. Оксиды $\mathrm{Cu}(\mathrm{I})$ и $\mathrm{Cu}(\mathrm{II})$, образованные на сплавах с вакансионно-разупорядоченным поверхностным слоем, характеризуются меньшими значениями ширины области пространственного заряда и дебаевской длины экранирования, но более высокими значениями показателя поглощения света и концентрации акцепторных дефектов. 


\section{СПИСОК ЛИТЕРАТУРЫ}

1. Pickering H. W. // J. Electrochem. Soc., 1968, vol. 115, № 2, pp. 143-147.

2. Маршаков И. К., Введенский А. В., Кондрашин В. Ю., Боков Г. А. Анодное растворение и селективная коррозия сплавов. Воронеж, Издательство ВГУ, 1988, 208 c.

3. Козадеров О. А., Введенский А. В. Массоперенос и фазообразование при анодном селективном растворении гомогенных сплавов. Воронеж, Научная книга, 2014, 288 c.

4. Гуревич Ю. Я., Плесков Ю. В. Фотоэлектрохимия полупроводников. М.: Наука, 1983, 312 с.

5. Bard A. J. Encyclopedia of Electrochemistry. V. 6: Semiconductor Electrodes and Photoelectrochemistry. Weinheim: Wiley - VCH, 2002, 51 p.

6. Bockris J. O’M., Khan S. U. M. Surface Electrochemistry: a Molecular Level Approach. Plenum Press, New York, 1993, $1014 \mathrm{p}$

7. Gerischer H. // Corros. Sci., 1989, vol. 29, № 2-3, pp. 257-266.

8. Кудряшов Д. А., Грушевская С. Н., Ганжа С. В., Введенский А. В. // Физикохимия поверхности и защчита материалов, 2009, т. 45, № 5, с. 451-460.

9. Кудряшов Д. А., Грушевская С. Н., Ганжа С. В., Введенский А. В. // Физикохимия поверхности и защита материалов, 2010, т. 46, № 1, с. 28-35.
10. Vvedenskii A. V., Grushevskaya S. N., Kudryashov D. A., Ganzha S. V. // J. Solid State Electrochem., 2010, vol. 14, № 8, pp. 1401-1413.

11. Муртазин М. М., Елисеев Д. С., Китаева Т. М., Грушевская С. Н., Введенский А. В. // Конденсированнье среды и межфазные гранииы, 2017, т. 19, № 1, с. 98-111.

12. Procaccini R., Vazquez M., Cere. S. // Electrochim. Acta., 2009, vol. 54, № 28, pp. 7324-7329.

13. Komura S., Furakawa H. Dynamics of Ordering Process in Condensed Matter, New-York, 1988, 574 p.

14. Clarebrough L. M., Loretto M. H. // Proc. R. Soc. Lond. A, 1960, vol. 257, pp. 326-327.

15. Елисеев Д. С., Грушевская С. Н., Абакумова Л. И. // Вестник Воронежского государственного университета. Серия: Химия. Биология. Фармация, 2014, № 1, с. 23-33.

16. Елисеев Д. С., Грушевская С. Н., Введенский А. В. // Вестник Тамбовского университета, 2013, т. 18, № 5, с. 2196-2200.

17. Грушевская С. Н., Елисеев Д. С., Введенский А. В. // Физикохимия поверхности и защита материалов, 2017, т. 53, № 2, с. 141-147.

18. Грушевская С. Н., Елисеев Д. С., Ганжа С. В., Введенский А. В. // Конденсированные среды и межфазные границы, 2013, т. 15, № 3, с. 253-265.

19. Самсонов Г. В. Физико-химические свойства окислов. М.: Металлургия, 1978, 472 с.

20. Collisi U., Strehblow H.-H. // J. Electroanal. Chem., 1986, vol. 210, p. 213-227.

\title{
PHOTOELECTROCHEMISTRY OF COPPER OXIDES ANODICALLY FORMED ON Cu-Zn ALLOYS
}

\author{
(C) 2017 M. Yu. Nesterova, S. N. Grushevskaya, A. V. Vvedenskii \\ Voronezh State University, 1 Universitetskaya sq., 394018 Voronezh, Russia \\ e-mail:sg@chem.vsu.ru
}

Received 04.07.2017

\begin{abstract}
This paper is aimed at revealing the influence of the vacancy defectiveness of the $\mathrm{Cu}-\mathrm{Zn}$ alloys surface layer on the kinetics of oxide formation and structure-sensitive properties of thin oxide films. Selective dissolution is used to generate superequilibrium vacancies in the alloys surface layer.

Preliminary selective dissolution with a monitored potential, charge, and concentration of vacancies was carried out in deoxygenated $0.01 \mathrm{M} \mathrm{HCl}+0.09 \mathrm{M} \mathrm{KCl}$. Subsequent anodic oxidation of $\mathrm{Cu}-\mathrm{Zn}$ alloys was carried out in deoxygenated $0.1 \mathrm{M} \mathrm{KOH}$ at the potentials of formation of $\mathrm{Cu}(\mathrm{I})$ and $\mathrm{Cu}(\mathrm{II})$ oxides. Chronoamperometry with synchronous registration of photocurrent and photopotential measurements in the open circuit were applied. The kinetics of anodic oxide formation is solid phase diffusion. The effective diffusion coefficient slightly increases with the potential of oxide formation and zinc concentration in the alloy.

It was established p-type conductivity for $\mathrm{Cu}(\mathrm{I})$ and $\mathrm{Cu}(\mathrm{II})$ oxides on $\mathrm{Cu}-\mathrm{Zn}$ alloys caused by prevalence of acceptor defects. Cathodic photocurrent increases in the course of polarization, reflecting
\end{abstract}


the thickening of oxide film. Positive photopotential increases over time after polarization is switched off, indicating the corrosion oxidation of copper from oxide-free electrode surface. The dependence of photocurrent and photopotential over time proves that the thickness of oxide films is less than the space charge region.

Quantitative processing of photocurrent vs. film thickness curves provides a set of structural-sensitive parameters, among which the light absorption coefficient $\alpha$, the concentration of acceptor defects $N_{\text {A }}$, space charge region $W$ and Debye length $L_{\mathrm{D}}$. Preliminary selective dissolution as well as the growth of zinc concentration in $\mathrm{Cu}-\mathrm{Zn}$ alloys results in an increase of $\alpha$ and $N_{\mathrm{A}}$ supplied with a decrease of $W$ and $L_{\mathrm{D}}$.

Keywords: copper-zinc alloys, copper oxides, selective dissolution, anodic oxide formation, photocurrent, photopotential.

\section{REFERENCES}

1. Pickering H. W. J. Electrochem. Soc., 1968, vol. 115, no. 2, pp. 143-147. DOI: 10.1149/1.2411048 Available at: http://jes.ecsdl.org/content/115/2/143.full.pdf+html

2. Marshakov I. K., Vvedenskii A. V., Kondrashin V. Yu., Bokov G. A. Anodic Dissolution and Selective Corrosion of Alloys. Voronezh, Voronezh State University Publ., 1988, 208 p. (in Russian)

3. Kozaderov O. A., Vvedenskii A. V. Mass Transfer and Phase Formation During Anodic Selective Dissolution of Homogeneous Alloys, Voronezh, Nauchnaya Kniga Publ., 2014, 288 p. (in Russian)

4. Gurevich Yu. Ya., Pleskov Yu. V. Fotoehlektrohimiya Poluprovodnikov. Moscow, Nauka Publ., 1983, 312 p. (in Russian)

5. Bard A. J. Encyclopedia of Electrochemistry. V. 6: Semiconductor Electrodes and Photoelectrochemistry, Weinheim, Wiley - VCH, 2002, 51 p.

6. Bockris J. O’M., Khan S. U. M. Surface Electrochemistry: a Molecular Level Approach. Plenum Press, New York, 1993, $1014 \mathrm{p}$.

7. Gerischer H. Corros. Sci., 1989, vol. 29, no. 2-3, pp. 257-266. DOI: 10.1016/0010-938X(89)90034-6 Available at: http://www.sciencedirect.com/science/article/ pii/0010938X89900346

8. Kudryashov D. A., Grushevskaya S. N., Ganzha S. V., Vvedenskii A. V. Protection of Metals and Physical Chemistry of Surfaces, 2009, vol. 45, no. 5, pp. 501-511. DOI: 10.1134/S2070205109050013 Available at: https://elibrary. ru/item.asp?id=15309505

9. Kudryashov D. A., Grushevskaya S. N., Ganzha S. V., Vvedenskii A. V. Protection of Metals and Physical Chemistry of Surfaces, 2010, vol. 46, no. 1, pp. 32-39. DOI: 10.1134/S2070205110010041 Available at: https://elibrary.ru/item.asp?id=15313507

10. Vvedenskii A. V., Grushevskaya S. N., Kudryashov D. A., Ganzha S. V. J. Solid State Electrochem., 2010, vol. 14, no. 8, pp. 1401-1413. DOI: 10.1007/s10008-0090952-9 Available at: https://link.springer.com/article/10.1007/s10008-009-0952-9/fulltext.html
11. Murtazin M. M., Eliseev D. S., Kitaeva T. M., Grushevskaya S. N., Vvedenskii A. V. Condensed Matter and Interphases, 2017, vol. 19, no. 1, pp. 98-111. Available at: http://www.kcmf.vsu.ru/resources/t_19_1_2017_011.pdf (in Russian)

12. Procaccini R., Vazquez M., Cere. S. Electrochim. Acta., 2009, vol. 54, no. 28, pp. 7324-7329. DOI: 10.1016/ j.electacta.2009.07.058 Available at: http://www.sciencedirect.com/science/article/pii/S0013468609009815

13. Komura S., Furakawa H. Dynamics of Ordering Process in Condensed Matter. New-York, 1988, 574 p.

14. Clarebrough L. M., Loretto M. H. Proc. R. Soc. Lond. A, 1960, vol. 257, pp. 326-327. DOI: 10.1098/ rspa.1960.0155 Available at: rspa.royalsocietypublishing. org

15. Eliseev D. S., Grushevskaya S. N., Abakumova L. I. Proceedings of Voronezh State University. Series: Chemistry. Biology. Pharmacy, 2014, no. 1, pp. 23-33. Available at: http://www.vestnik.vsu.ru/pdf/chembio/2014/01/201401-05.pdf

16. Eliseev D. S., Grushevskaya S. N., Vvedenskii A. V. Vestnik Tambov State University, 2013, vol. 18, no. 5, pp. 2196-2200. Available at: https://elibrary.ru/item. asp?id=20141615 (in Russian)

17. Grushevskaya S. N., Eliseev D. S., Vvedenskii A. V. Protection of Metals and Physical Chemistry of Surfaces, 2017, vol. 53, no. 2, pp. 141-147 Available at: https://elibrary.ru/item.asp?id=28918437

18. Grushevskaya S. N., Eliseev D. S., Ganzha S. V., Vvedenskii A. V. Condensed Matter and Interphases, 2013, vol. 15, no. 3, pp. 253-265. Available at: http://www.kcmf. vsu.ru/resources/t_15_3_2013_006.pdf (in Russian)

19. Samsonov G. V. Physico-Chemical Properties of Oxides. Moscow, Metallurgiya Publ., 1978, 472 p. (in Russian)

20. Collisi U., Strehblow H.-H. J. Electroanal. Chem., 1986, vol. 210, p. 213-227. DOI: 10.1016/00220728(86)80573-3 Available at: http://www.sciencedirect. com/science/article/pii/0022072886805733 


\section{М. Ю. НЕСТЕРОВА, С. Н. ГРУШЕВСКАЯ, А. В. ВВЕДЕНСКИЙ}

Нестерова Мария Юрьевна - магистрант второго года обучения химического факультета, Воронежский государственный университет; тел.: +7(473) 2208538, e-mail: nesterovamarija18@gmail.com

Грушевская Светлана Николаевна - к. х. н., доцент, доцент кафедры физической химии, Воронежский государственный университет; тел.: +7(920) 4067144, e-mail: sg@chem.vsu.ru

Введенский Александр Викторович - д. х. н., профессор, заведующий кафедрой физической химии, Воронежский государственный университет; тел.: +7(473) 2208546, e-mail: alvved@chem.vsu.ru
Nesterova Marija Yu. - Second-year Student of Magister Program on Chemistry Faculty, Voronezh State University; ph.: +7(473) 2208538, e-mail: nesterovamarija18@ gmail.com

Grushevskaya Svetlana N. - Cand. Sci. (Chem.), Associate Professor of Physical Chemistry Department, Voronezh State University; ph.: +7(920) 4067144, e-mail: sg@chem.vsu.ru

Vvedenskii Alexander V. - Dr. Sci. (Chem.), Professor, Chief of Physical Chemistry Department, Voronezh State University; ph.: +7(473) 2208546, e-mail: alvved@ chem.vsu.ru 